\title{
Nível de atividade física de estudantes de educação física no Brasil: uma revisão sistemática
}

\author{
Physical activity level of physical education students in Brazil: a systematic review
}

AUTORES
Vítor Tavares da Silva $^{1}$ (D)
Lorena Rodrigues Silva $^{1}$ (D)
Vinicius Guadalupe Barcelos Oliveira $^{1}$ (D)
Bianca Pagel Ramson $^{1}$ (D)
Eduardo Lucia Caputo $^{1}$ (D)
Marcelo Cozzensa da Silva ${ }^{1}$ (D)
1 Universidade Federal de Pelotas, Escola Superior
de Educação Física, Pelotas, Rio Grande do Sul,
Brasil.
CONTATO
Vítor Tavares da Silva
vitortavarees@outlook.com
Rua Luís de Camões, 625, Três Vendas,
Pelotas, Rio Grande do Sul, Brasil.
CEP: 96055-630.
DOI

$10.12820 /$ rbafs.26e0197

\section{(cc) BY}

Este trabalho está licenciado com uma Licença Creative Commons - Atribuição 4.0 Internacional.

\begin{abstract}
RESUMO
Este estudo teve como objetivo identificar os níveis de atividade física de estudantes do curso de Educação Física no Brasil. Metodologicamente tratou-se de uma revisão sistemática da literatura, a qual foi estruturada a partir das recomendações do Preferred Reporting Items for Systematic Reviews and Meta-Analyses (PRISMA). As buscas foram realizadas nas bases de dados LILACS, PubMed, SciELO e SPORTDiscus. Os artigos originais incluídos foram apenas aqueles que apresentavam resultados quanto ao nível de atividade física de estudantes do curso de graduação em Educação Física, no Brasil, bem como utilizaram instrumentos validados. A partir dos critérios de inclusão, 15 estudos passaram pelas etapas de análise. A maioria das investigações ocorreram na região Sul do Brasil, utilizou o International Physical Activity Questionnaire (IPAQ) para aferir a atividade física e as recomendações da Organização Mundial da Saúde (OMS) na avaliação do nível de prática. Todos os estudos apresentaram uma alta prevalência de estudantes fisicamente ativos, variando entre 71,5\% e 100\%. Pode-se concluir que os estudantes do curso de Educação Física têm se mostrado enquanto uma população predominantemente ativa no Brasil.
\end{abstract}

Palavras-chave: Educação física; Ensino superior; Alunos; Atividade física.

ABSTRACT

The aim of this study was to review the physical activity levels of Physical Education graduate students in Brazil. Methodologically this study is a systematic review of the literature, since it was elaborated based the recommendations for the Systematic Reviews and Meta-Analyzes (PRISMA). Searches were performed in LILACS, PubMed, SciELO and SPORTDiscus databases. Articles which presented results regarding physical activity levels from Physical Education graduate students, as well as have used validated instruments, were included. A final number of 15 studies were included. Most of the surveys were conducted on Southern Brazil, the International Physical Activity Questionnaire (IPAQ) to verify physical activity and the World Health Organization (WHO) recommendations for assessing the physical activity level. All studies presented a high prevalence of physically active students. It can be concluded that Physical Education graduate students have been shown to be a predominantly active population in Brazil.

Keywords: Physical education; College; Students; Physical activity.

\section{Introdução}

A atividade física é reconhecida como fator protetor para uma série de doenças crônicas não transmissíveis ${ }^{1,2}$. Contudo, nos últimos anos, é possível observar uma redução preocupante dos níveis populacionais de atividade física ${ }^{3-5}$. Especificamente no Brasil, segundo dados da Pesquisa Nacional por Amostra de Domicílio, no ano de $2015,87,4 \%$ dos indivíduos não praticaram qualquer tipo de atividade física regular ${ }^{6}$. Outras investigações no país também alertam para altos níveis de inatividade física entre adultos e adolescentes ${ }^{7-9}$. No caso dos adolescentes, a literatura científica tem tentado identificar a possível relação entre a prática de atividade física realizada nessa fase do desenvolvimento e a continuidade da mesma na fase adulta ${ }^{10,11}$.
Para muitos estudantes, esse período de transição maturacional coincide com o ingresso no ensino superior. Esta etapa tende a ser muito importante na consolidação de hábitos saudáveis, entre eles a prática de atividades físicas ${ }^{12}$. Neste sentido, tendo em vista que o ingresso na faculdade é um evento marcado por transformações nas relações sociais, hábitos e comportamentos dos estudantes ${ }^{13}$, torna-se importante a realização de estudos a fim de investigar desfechos relacionados ao estilo de vida nessa população.

Neste contexto, dentre a população discente universitária brasileira, tem-se destacado um baixo percentual de alunos ativos fisicamente ${ }^{14}$. $\mathrm{Na}$ ênfase de cursos dedicados a conhecimentos específicos da saúde, a Educação Física se destaca no campo de estudo dos benefícios da 
prática de atividade física. Estudos indicam que dentre a população universitária, os alunos do curso de Educação Física geralmente são mais ativos que os demais cur$\operatorname{sos}^{15-17}$, apresentando hábitos mais saudáveis em seu cotidiano devido em grande parte aos conhecimentos acerca da atividade física e saúde disseminados no curso ${ }^{14,18,19}$.

Contudo, a literatura científica ainda não conta com estudos que sintetizem os resultados já obtidos em relação aos níveis de atividade física de estudantes dos cursos de graduação em Educação Física brasileiros. Informações acerca do delineamento utilizado, do método de avaliação, assim como do nível de atividade física alcançado por esses estudantes, são importantes para compreender como se dá o padrão de atividade física nessa população. $\mathrm{O}$ preenchimento dessa lacuna auxilia na demarcação do contexto desta população que assume como perspectiva uma atuação profissional baseada na atividade física, e, portanto, com desdobramentos sociais. O objetivo desta revisão foi identificar o nível de atividade física de estudantes do curso de Educação Física no Brasil.

\section{Métodos}

Este estudo se caracteriza como uma revisão sistemática da literatura, a qual foi estruturada a partir das diretrizes do Preferred Reporting Items for Systematic Reviews and Meta-Analyses (PRISMA) ${ }^{20}$, e registrada na base PROSPERO (CRD42020118584). As buscas foram realizadas nas seguintes bases de dados: LILACS, PubMed, SciELO e SPORTDiscus.

O processo de busca dos dados foi definido e refinado a partir da estratégia do acrônimo "PICOS"21, determinando os descritores ou termos baseados nas sugestões dos Descritores em Ciências da Saúde (DeCS) e do Medical Subject Headings (MeSH), considerando a população, a exposição e desfecho empregados de acordo com as seguintes combinações: "students" AND "physical education" AND “course" OR “degree” OR "graduate" OR "college" OR "university” AND "physical activity" OR “active" OR “inactive” OR "sedentary”.

As buscas foram realizadas nas línguas inglesa e portuguesa e foram investigadas todas as referências disponíveis na literatura até o mês de março de 2020. Dois investigadores independentes (VTS e VGBO) conduziram as buscas e, em caso de discordância um terceiro investigador foi consultado (LRS).

Foram incluídos no processo de busca artigos originais que apresentassem resultados sobre o nível de atividade física de estudantes do curso de graduação em Educação Física obtidos por meio de instrumentos validados. Como critério de exclusão foram adotados os seguintes parâmetros: artigos de opinião, cartas ao editor, livros, capítulos de livros, relatórios de pesquisa, dissertações e teses, artigos contendo informação sobre o nível de atividade física de estudantes do curso de Educação Física fora do Brasil.

A triagem inicial consistiu na leitura dos títulos, exclusão de artigos repetidos e seleção daqueles que atendiam aos critérios de inclusão. Posteriormente, procedeu-se a leitura do resumo e, em seguida, leitura na íntegra daqueles que atendiam a todos os critérios. Ao final do processo de leitura dos textos completos, foi conduzida busca manual na lista de citações dos estudos incluídos.

A qualidade dos estudos foi avaliada por dois revisores independentes (VTS e VGBO). Em caso de discordância, um terceiro revisor foi consultado a fim de obter-se consenso (ELC). A Nervcastle-Ottawa Scale $(\mathrm{NOS})^{22}$ foi utilizada para avaliar a qualidade dos estudos incluídos. A escala NOS é usada para avaliar a qualidade de estudos longitudinais. Essa escala possui oito itens relacionados à seleção, comparabilidade e resultado dos estudos. Para estudos longitudinais, foi utilizada a escala NOS original. Para estudos transversais, foi realizada uma adaptação da escala NOS de estudos de coorte, com base na adaptação publicada anteriormente de Herzog et al. ${ }^{23}$.

Neste último, a escala foi composta por sete itens relacionados também à seleção, comparabilidade e resultado. $\mathrm{Na}$ NOS, cada item recebe uma estrela se o estudo for classificado como de alta qualidade, exceto o componente de comparabilidade que pode receber duas estrelas. Compreende-se, nesta revisão, que escores de 8 a 10 podem ser classificados como de alta qualidade metodológica, 5 a 7 como de média qualidade, e 0 a 4 como de baixa qualidade.

Os dados de cada um dos estudos foram tabulados em uma planilha gerada no software Microsoft Excel ${ }^{\circledR}\left(\mathrm{pa}^{-}\right.$ cote Microsoft Office ${ }^{\circledR}$ versão 2013, desenvolvido pela Microsoft ${ }^{\circledR}$ ) contendo informações sobre os autores, ano de publicação, estado onde se realizou o estudo, características e tamanho amostral, delineamento da pesquisa, instrumentos utilizados e principais achados relativos à ocorrência do desfecho (nível de atividade física).

\section{Resultados}

Ao todo, foram identificados 238 estudos, sendo excluídos 209 estudos por não ter relação com o tema, ser 
repetido ou serem realizados fora do Brasil. Diante disso, 29 estudos foram lidos na íntegra, sendo 14 excluídos por envolverem a mesma amostra, não mensurar o nível de atividade física e/ou não utilizarem instrumentos válidos. Por fim, 15 artigos atenderam aos critérios de inclusão e foram incluídos. $\mathrm{O}$ processo de seleção dos artigos está detalhado no fluxograma (Figura 1).

Com relação à qualidade de evidência, cinco estudos apresentaram escore máximo na escala ${ }^{18,24,25,26,27}$,

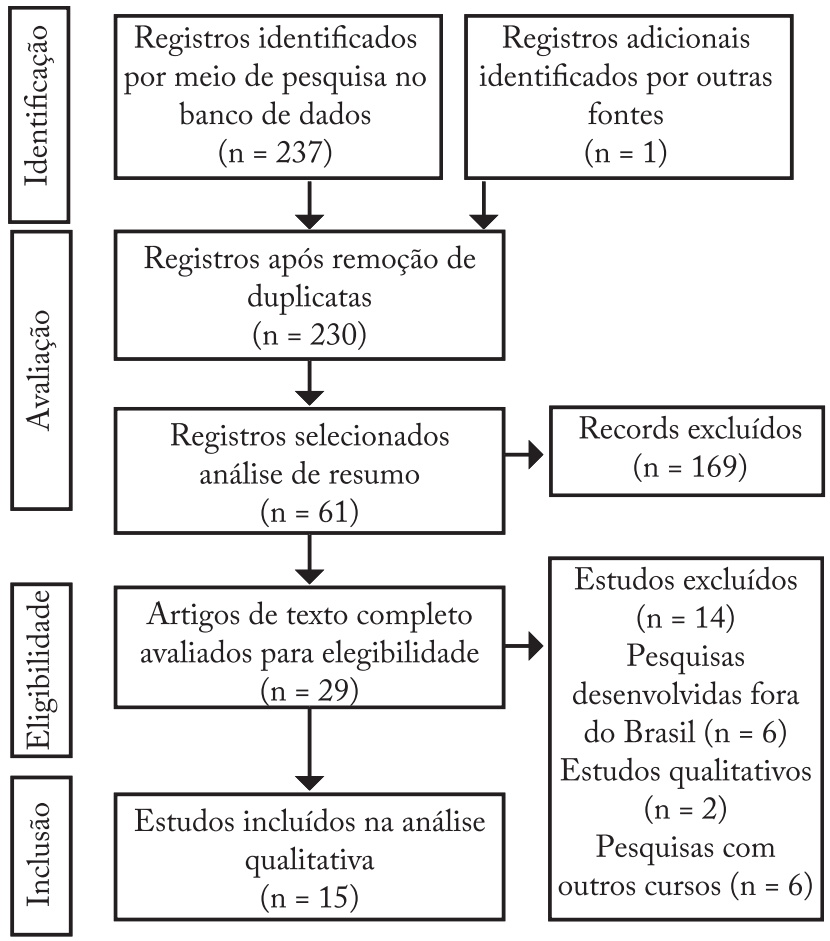

Figura 1 - Fluxograma de seleção dos estudos incluídos. cinco obtiveram 9 pontos ${ }^{17-19,28,29,30}$, quatro tiveram 8 pontos $^{31,32,33,34}$ e um obteve 6 pontos $^{16}$ (Tabela 1 ).

A Tabela 2 descreve as características dos estudos que compuseram a revisão. A maioria das investigações foi realizada na região Sul do Brasil $(60,0 \%)$ (nove estudos ${ }^{17,19,25-27,30-33}$ ), seguida das regiões Sudeste (três estudos $\left.^{16,24,29}\right)(20,0 \%)$, região Nordeste (dois estudos ${ }^{18,28}$ $(13,3 \%)$ e região centro-oeste (um estudo $\left.{ }^{34}\right)(6,7 \%)$. As pesquisas foram descritas por estado a fim de preservar as identidades das instituições e amostras (estratégia adotada por parte dos estudos incluídos). Quanto ao ano de publicação, quatro estudos foram conduzidos até $2010^{16,17,19,25}$, seis entre 2011 e $2015^{18,26-29,32}$ e cinco a partir de $2016^{24,30,31,33,34}$.

Todos os estudos que compuseram a revisão apresentaram caráter observacional, sendo 14 utilizando delineamento transversal ${ }^{16-19,24-27-30,32-34} \mathrm{e}$ apenas $\mathrm{um}^{31}$ longitudinal. Quanto ao número de estudantes investigados, o estudo com menor número amostral foi o de Freitas et al. ${ }^{27}$ com 38 participantes, e o maior foi o de Monteiro et al. ${ }^{34}$ com 909. Três estudos investigaram menos de 100 estudantes ${ }^{17,27,31}$, cinco estudos tinham entre 114 e 199 estudantes ${ }^{26,28,29,30,33}$ e outros sete com 200 estudantes ou mais ${ }^{16,18,19,24,25,32,34}$.

Em relação ao sexo, sete estudos apresentaram maior número de participantes do sexo masculino ${ }^{18,29,26,27,31-33}$, cinco do feminino ${ }^{16,19,24,25,34}$, um teve número idênti$\mathrm{co}^{30}$, e dois não relataram o número de estudantes do curso de Educação Física por sexo ${ }^{17,28}$. Cinco estudos não apresentaram os dados do nível de atividade física estratificados por sexo ${ }^{17,25,28,33,34}$. Entre os estudos que

Tabela 1 - Descrição da qualidade dos estudos avaliados pela escala Newcastle-Ottawa Scale (NOS) ( $\mathrm{n}=15)$

\begin{tabular}{|c|c|c|c|c|c|c|c|c|c|}
\hline \multirow{2}{*}{$\begin{array}{l}\text { Estudo } \\
\text { (primeiro autor) }\end{array}$} & \multirow{2}{*}{$\begin{array}{l}\text { Desenho do } \\
\text { Estudo }\end{array}$} & \multicolumn{2}{|l|}{ Seleção } & \multicolumn{2}{|c|}{ Comparabilidade } & \multicolumn{2}{|c|}{ Resultado } & \multicolumn{2}{|c|}{ Pontuação } \\
\hline & & $\begin{array}{c}\text { Representatividade } \\
\text { de amostra }\end{array}$ & $\begin{array}{l}\text { Tamanho } \\
\text { da amostra }\end{array}$ & $\begin{array}{c}\text { Não } \\
\text { respondentes }\end{array}$ & $\begin{array}{l}\text { Verificação da } \\
\text { exposição }\end{array}$ & $\begin{array}{c}\text { Baseado no } \\
\text { desenho e análise }\end{array}$ & $\begin{array}{l}\text { Avaliação do } \\
\text { resultado }\end{array}$ & $\begin{array}{c}\text { Teste } \\
\text { estatístico }\end{array}$ & Total \\
\hline Silva et al. ${ }^{16}$ & Transversal & $*$ & - & - & * & * & ** & * & 6 \\
\hline Mielke et al. ${ }^{17}$ & Tranversal & $* *$ & $*$ & $*$ & $*$ & * & ** & * & 9 \\
\hline Silva $^{18}$ & Tranversal & $* *$ & * & - & $*$ & $* *$ & $* *$ & * & 9 \\
\hline Bielemann et al. ${ }^{19}$ & Tranversal & $* *$ & $*$ & $*$ & $*$ & * & ** & $*$ & 9 \\
\hline Melo et al. ${ }^{24}$ & Tranversal & $* *$ & $*$ & * & $*$ & $* *$ & $* *$ & * & 10 \\
\hline Rech et al. ${ }^{25}$ & Tranversal & $* *$ & $*$ & $*$ & $*$ & $* *$ & $* *$ & $*$ & 10 \\
\hline Vargas et al. ${ }^{26}$ & Tranversal & $* *$ & $*$ & * & $*$ & $* *$ & $* *$ & $*$ & 10 \\
\hline Freitas et al. ${ }^{27}$ & Tranversal & $* *$ & $*$ & $*$ & $*$ & $* *$ & $* *$ & $*$ & 10 \\
\hline Costa et al. ${ }^{28}$ & Tranversal & $* *$ & $*$ & $*$ & $*$ & * & $* *$ & $*$ & 9 \\
\hline Miranda et al. ${ }^{29}$ & Tranversal & $* *$ & $*$ & $*$ & $*$ & * & $* *$ & * & 9 \\
\hline Lima et al. ${ }^{30}$ & Tranversal & $* *$ & $*$ & $*$ & $*$ & * & $* *$ & $*$ & 9 \\
\hline Santos et al. ${ }^{31}$ & Longitudinal & $*$ & $*$ & - & $*$ & $* *$ & $* *$ & * & 8 \\
\hline Ferrari et al. ${ }^{32}$ & Tranversal & $*$ & $*$ & $*$ & $*$ & $*$ & $* *$ & $*$ & 8 \\
\hline Mieira et al. ${ }^{33}$ & Tranversal & * & $*$ & $*$ & $*$ & $*$ & $* *$ & $*$ & 8 \\
\hline Monteiro et al. ${ }^{34}$ & Tranversal & $*$ & $*$ & $*$ & $*$ & $*$ & $* *$ & $*$ & 8 \\
\hline
\end{tabular}


Tabela 2 - Estudos sobre nível de atividade física de estudantes de Educação Física no Brasil segundo o autor, ano, local, amostra, faixa etária, seleção amostral, delineamento, instrumento e principais resultados $(n=15)$

\begin{tabular}{|c|c|c|c|c|c|c|c|}
\hline Autor (Ano) & Local & $\begin{array}{l}\text { Amostra total (por } \\
\text { Sexo) }\end{array}$ & $\begin{array}{l}\text { Faixa etária } \\
\text { (anos) }\end{array}$ & Seleção da amostra & Delineamento & Instrumento & Principais resultados* \\
\hline $\begin{array}{l}\text { Silva } \\
(2011)^{18}\end{array}$ & Sergipe & $\begin{array}{l}217 \\
(98 \mathrm{~F} \text { e } 119 \mathrm{M})\end{array}$ & $\leq 20(59,5 \%)$ & $\begin{array}{l}\text { Realizada pelo método } \\
\text { aleatório simples, através de } \\
\text { sorteio. }\end{array}$ & Transversal & IPAQ (curto) & $\begin{array}{l}89,4 \% \text { ativos e } 10,6 \% \\
\text { pouco ativos }\end{array}$ \\
\hline $\begin{array}{l}\text { Costa et al. } \\
(2015)^{28}\end{array}$ & Paraíba & 199 & $21,3 \pm 4$ & $\begin{array}{l}\text { Todos os estudantes } \\
\text { matriculados no primeiro, } \\
\text { segundo, sétimo e oitavo } \\
\text { semestres foram incluídos. }\end{array}$ & Transversal & IPAQ (curto) & $84,4 \%$ ativos \\
\hline $\begin{array}{l}\text { Melo et al. } \\
(2016)^{24}\end{array}$ & $\begin{array}{l}\text { Espírito } \\
\text { Santo }\end{array}$ & $\begin{array}{l}285 \\
(143 \mathrm{~F} \text { e } 142 \mathrm{M})\end{array}$ & $23,8 \pm 5$ & $\begin{array}{l}\text { Amostragem estratificada em } \\
\text { todos os períodos dos cursos. } \mathrm{O} \\
\text { artigo não específica como foi a } \\
\text { escolha direta dos participantes. }\end{array}$ & Transversal & IPAQ (curto) & $\begin{array}{l}86 \% \text { muito ativos e } \\
\text { ativos }\end{array}$ \\
\hline $\begin{array}{l}\text { Miranda et } \\
\text { al. }(2013)^{29}\end{array}$ & $\begin{array}{l}\text { Minas } \\
\text { Gerais }\end{array}$ & $\begin{array}{l}197 \\
(93 \mathrm{~F} \text { e } 104 \mathrm{M})\end{array}$ & $21,2 \pm 2.3$ & $\begin{array}{l}\text { Todos os estudantes } \\
\text { matriculados do primeiro ao } \\
\text { oitavo período do curso. }\end{array}$ & Transversal & IPAQ (curto) & $\begin{array}{l}41,1 \% \text { muito ativos; } \\
42,6 \text { ativos; } 12,3 \% \\
\text { insuficientemente } \\
\text { ativo } \mathrm{A} ; 3,0 \% \\
\text { insuficientemente } \\
\text { ativos B; } 1,0 \% \\
\text { sedentários }\end{array}$ \\
\hline $\begin{array}{l}\text { Monteiro et } \\
\text { al. }(2019)^{34}\end{array}$ & $\begin{array}{l}\text { Distrito } \\
\text { Federal }\end{array}$ & $\begin{array}{l}909 \\
(518 \mathrm{~F} \text { e } 385 \mathrm{M})\end{array}$ & $20-29(67,6 \%)$ & $\begin{array}{l}\text { Foi composta por estudantes do } \\
\text { primeiro ao oitavo semestre que } \\
\text { estavam em suas salas de aula } \\
\text { no dia de coleta de dados. }\end{array}$ & Transversal & $\begin{array}{l}\text { IPAQ } \\
\text { (longo) }\end{array}$ & $71,5 \%$ ativos \\
\hline $\begin{array}{l}\text { Rech et al. } \\
(2010)^{25}\end{array}$ & Paraná & $\begin{array}{l}294 \\
(158 \mathrm{~F} \text { e } 136 \mathrm{M})\end{array}$ & $21,9 \pm 4,7$ & $\begin{array}{l}\text { Selecionado todos os estudantes } \\
\text { regularmente matriculados. }\end{array}$ & Transversal & IPAQ (curto) & $\begin{array}{l}\text { Nível de AF: } 5,1 \% \\
\text { alto; } 92,1 \% \text { moderada; } \\
2,8 \% \text { baixa }\end{array}$ \\
\hline $\begin{array}{l}\text { Vargas et al. } \\
(2015)^{26}\end{array}$ & Paraná & $\begin{array}{l}189 \\
(93 \mathrm{~F} \text { e } 96 \mathrm{M})\end{array}$ & $33,5 \pm 8,3$ & $\begin{array}{l}\text { Realizada pelo método } \\
\text { aleatório simples, através de } \\
\text { sorteio dos registros acadêmicos } \\
\text { dos estudantes. }\end{array}$ & Transversal & $\begin{array}{l}\text { IPAQ } \\
\text { (longo) }\end{array}$ & $79,8 \%$ ativos \\
\hline $\begin{array}{l}\text { Lima et al. } \\
(2017)^{30}\end{array}$ & $\begin{array}{l}\text { Rio } \\
\text { Grande } \\
\text { do Sul }\end{array}$ & $\begin{array}{l}114 \\
(57 \mathrm{~F} \text { e } 57 \mathrm{M})\end{array}$ & - & $\begin{array}{l}\text { Foram selecionados de forma } \\
\text { aleatória. }\end{array}$ & Transversal & IPAQ (curto) & $\begin{array}{l}89 \% \text { muito ativos ou } \\
\text { ativos }\end{array}$ \\
\hline $\begin{array}{l}\text { Mielke et al. } \\
(2010)^{17}\end{array}$ & $\begin{array}{l}\text { Rio } \\
\text { Grande } \\
\text { do Sul }\end{array}$ & 67 & $22,6 \pm 7,3$ & $\begin{array}{l}\text { Sorteio aleatório dos cursos } \\
\text { dentro de cada área pré- } \\
\text { selecionada e seleção de todos } \\
\text { os estudantes ingressantes nos } \\
\text { cursos sorteados. }\end{array}$ & Transversal & $\begin{array}{l}\text { IPAQ } \\
\text { (longo) }\end{array}$ & $95,1 \%$ ativos \\
\hline $\begin{array}{l}\text { Santos et al. } \\
(2017)^{31}\end{array}$ & $\begin{array}{l}\text { Rio } \\
\text { Grande } \\
\text { do Sul }\end{array}$ & $\begin{array}{l}81 \\
(38 \mathrm{~F} \text { e } 43 \mathrm{M})\end{array}$ & - & $\begin{array}{l}\text { Foram selecionados todos os } \\
\text { estudantes ingressantes } \\
\text { ano de 2014, os quais foram } \\
\text { acompanhados por um ano com } \\
\text { realização de duas avaliações } \\
\text { (baseline e um ano após) }\end{array}$ & Longitudinal & $\begin{array}{l}\text { IPAQ } \\
\text { (longo) }\end{array}$ & $\begin{array}{l}\text { AF total } 2014: 85,4 \% \\
\text { ativos; AF total } 2015: \\
84,3 \%\end{array}$ \\
\hline $\begin{array}{l}\text { Bielemann } \\
\text { et al. } \\
(2007)^{19}\end{array}$ & $\begin{array}{l}\text { Rio } \\
\text { Grande } \\
\text { do Sul }\end{array}$ & $\begin{array}{l}221 \\
(116 \mathrm{~F} \text { e } 105 \mathrm{M})\end{array}$ & $22,2 \pm 3,1$ & $\begin{array}{l}\text { Selecionado todos os estudantes } \\
\text { regularmente matriculados e } \\
\text { frequentando as aulas.. }\end{array}$ & Transversal & $\begin{array}{l}\text { IPAQ } \\
\text { (longo) }\end{array}$ & $74,2 \%$ ativos no lazer \\
\hline $\begin{array}{l}\text { Freitas et al. } \\
(2013)^{27}\end{array}$ & $\begin{array}{l}\text { Rio } \\
\text { Grande } \\
\text { do Sul }\end{array}$ & $\begin{array}{l}38 \\
(12 \mathrm{~F} \text { e } 26 \mathrm{M})\end{array}$ & $21,1 \pm 2,2$ & $\begin{array}{l}\text { Foram selecionados todos } \\
\text { os estudantes que cursaram } \\
\text { a disciplina de Fisiologia } \\
\text { do Exercício II no segundo } \\
\text { semestre no ano de } 2012 \text { e } \\
\text { presentes no dia da coleta. }\end{array}$ & Transversal & $\begin{array}{l}\text { IPAQ } \\
\text { (longo) }\end{array}$ & $\begin{array}{l}100 \% \text { foram } \\
\text { considerados } \\
\text { fisicamente ativos } \\
\text { (IPAQ auto } \\
\text { preenchido); 92,1\% } \\
\text { fisicamente ativos } \\
\text { (IPAQ entrevista). }\end{array}$ \\
\hline $\begin{array}{l}\text { Ferrari et al. } \\
(2013)^{32}\end{array}$ & $\begin{array}{l}\text { Santa } \\
\text { Catarina }\end{array}$ & $\begin{array}{l}236 \\
(109 \mathrm{~F} \text { e } 127 \mathrm{M})\end{array}$ & $<20(56,8 \%)$ & $\begin{array}{l}\text { Foram convidados para } \\
\text { participar do estudo todos os } \\
\text { estudantes matriculados nos } \\
\text { cursos de EF. }\end{array}$ & Transversal & IPAQ (curto) & $\begin{array}{l}21,5 \% \text { mais ativos; } \\
25,3 \% \text { intermediário } \\
\text { ativos II; } 24,5 \% \\
\text { intermediário ativos I; } \\
28,3 \% \text { menos ativos }\end{array}$ \\
\hline
\end{tabular}


... continuação Tabela 2 - Estudos sobre nível de atividade física de estudantes de Educação Física no Brasil segundo o autor, ano, local, amostra, faixa etária, seleção amostral, delineamento, instrumento e principais resultados $(\mathrm{n}=15)$

\begin{tabular}{|c|c|c|c|c|c|c|c|}
\hline Autor (Ano) & Local & $\begin{array}{l}\text { Amostra total (por } \\
\text { Sexo) }\end{array}$ & $\begin{array}{l}\text { Faixa etária } \\
\text { (anos) }\end{array}$ & Seleção da amostra & Delineamento & Instrumento & Principais resultados ${ }^{*}$ \\
\hline $\begin{array}{l}\text { Meira et al. } \\
(2019)^{33}\end{array}$ & $\begin{array}{l}\text { Santa } \\
\text { Catarina }\end{array}$ & $\begin{array}{l}115 \\
(36 \mathrm{~F} \mathrm{79M})\end{array}$ & $25,0 \pm 6,2$ & $\begin{array}{l}\text { Foram selecionados todos os } \\
\text { estudantes matriculados nos } \\
\text { cursos de EF acima de } 18 \text { anos. }\end{array}$ & Transversal & $\begin{array}{l}\text { Escala de } \\
\text { Práticas no } \\
\text { Lazer (EPL) }\end{array}$ & $\begin{array}{l}91,3 \% \text { fisicamente } \\
\text { ativos no lazer. }\end{array}$ \\
\hline $\begin{array}{l}\text { Silva et al. } \\
(2007)^{16}\end{array}$ & $\begin{array}{l}\text { Minas } \\
\text { Gerais }\end{array}$ & $\begin{array}{l}194 \\
(94 \mathrm{~F} \text { e } 100 \mathrm{M}) \\
\text { amostra } 1^{\circ} \text { ao } 8^{\circ} \text { ano } \\
\text { curso EF; } 280 \\
(163 \mathrm{~F} \text { e } 117 \mathrm{M}) \\
\text { amostra total }\end{array}$ & $\begin{array}{l}21,2 \pm 2,7 \\
\text { (estudantes EF); } \\
19,7 \pm 2,6 \\
\text { (quatro cursos } \\
\text { incluído a EF) }\end{array}$ & $\begin{array}{l}\text { A forma de escolha dos } \\
\text { indivíduos não foi descrita no } \\
\text { artigo }\end{array}$ & Transversal & $\begin{array}{l}\text { IPAQ } \\
\text { (curto) }\end{array}$ & $\begin{array}{l}92 \% \text { muito ativos ou } \\
\text { ativos }\end{array}$ \\
\hline
\end{tabular}

$\mathrm{F}$ = feminino; $\mathrm{M}=$ masculino; $\mathrm{AF}$ = Atividade Física $\mathrm{EF}$ = Educação Física; IPAQ = International Physical Activity Questionnaire; ${ }^{*}$ Foram considerados ativos aqueles indivíduos que obtiveram 150 minutos ou mais de prática de atividade física semanal; - = Informações não são contempladas nos artigos incluídos.

apresentaram este dado, a maioria relatou resultados indicando os participantes do sexo masculino como os mais ativos $18,19,24,27,29,31,32$.

Considerando as diferentes nomenclaturas utilizadas nos artigos para classificar os estudantes de Educação Física como fisicamente ativos ("muito ativo", "ativo", "comportamento ativo", "suficientemente ativo", "nível de atividade física alto" e "nível de atividade física moderado"), o estudo que apresentou menor prevalência de estudantes fisicamente ativos foi o de Monteiro et al. ${ }^{34}(71,5 \%)$. A prevalência média e a mediana dos estudantes fisicamente ativos encontradas nos 12 estudos que avaliaram os níveis de atividade física totais foram, respectivamente, de 88,0\% e 87,5\%. Já entre as pesquisas dedicadas à apenas a dimensão de lazer ${ }^{19,33}$, destaca-se uma média de $82,8 \%$ de fisicamente ativos.

Dos quinze estudos investigados, quatorze utilizaram o Internacional Physical Activity Questionnaire (IPAQ) como instrumento para medir o nível de atividade física dos estudantes. Dentre estes, oito ${ }^{16,18,24,25,28-}$ ${ }^{30-32}$ utilizaram a versão curta do instrumento, apresentando prevalências de atividade física total que variaram entre $83,7 \%{ }^{29}$ e $97,2 \%{ }^{25}$. Os estudos de Bielemann et al. ${ }^{19}$ e Meira et al. ${ }^{33}$ avaliaram somente a prática de atividade física no lazer, sendo que Bielemann et al. ${ }^{19}$ utilizou o domínio de lazer da versão longa do IPAQ e Meira et al. ${ }^{33}$ a Escala de Práticas de Lazer para tal finalidade. Os estudos encontraram prevalências de $74 \%{ }^{19}$ e $91,3 \%{ }^{33}$ de atividade física suficiente no lazer entre os estudantes. Os demais estudos que utilizaram a versão longa do IPAQ ${ }^{17,19,26,27,31,34}$ avaliaram a atividade física total, a qual variou entre $71,5 \% \%^{34}$ e $100 \%{ }^{27}$.

Seis artigos ${ }^{16,19,28,29,31,33}$ analisaram a relação entre ano do curso de graduação e o nível de atividade física dos estudantes. $\mathrm{O}$ artigo de Bielemann et al. ${ }^{19}$ foi o úni- co a encontrar associação significativa entre essas variáveis, sendo que estudantes do sexo feminino mostraram tendência de queda nos níveis de atividade física suficiente conforme havia progressão no ano de curso.

Apenas os estudos de Mielke et al. ${ }^{17}$ e Santos et al. ${ }^{31}$ conduziram, além da avaliação da atividade física total dos estudantes, uma análise da atividade física estratificada segundo os domínios da atividade física, sendo que os avaliados foram o de lazer e deslocamento. $\mathrm{O}$ estudo de Mielke e colaboradores ${ }^{17}$ encontrou que estudantes dos cursos de bacharelado e licenciatura em Educação Física apresentaram maiores prevalências de atividade física de lazer quando comparado aos demais cursos investigados em uma universidade pública do sul do Brasil.

\section{Discussão}

O presente estudo evidenciou que a maioria das investigações que avaliaram o nível de atividade física em estudantes de Educação Física, no Brasil, foram realizados nas regiões Sul e Sudeste. Ainda, do ponto de vista metodológico quase a totalidade dos estudos incluídos utilizaram o delineamento transversal. Os resultados apontam para uma alta prevalência de atividade física suficiente em alunos de graduação em Educação Física.

O debate acerca do ambiente universitário vem destacando-se como um lugar apropriado para promoção de saúde. Contudo, esse ambiente tem sido pouco explorado a nível nacional no que se refere a estratégias para promoção de saúde institucional, sendo que o estilo de vida e a relação com saúde dos universitários ficam à mercê das particularidades de cada curso, bem como a variáveis externas à universidade ${ }^{35}$.

A qualidade geral dos estudos, avaliada pela escala NOS, pode ser considerada elevada, sendo que as inves- 
tigações que apresentaram menor pontuação obtiveram oito pontos. Entretanto, novos estudos com desenho longitudinal são necessários a fim de se investigar o padrão de atividade física nessa população ao longo de sua vida acadêmica, visto que somente um estudo que compõe essa revisão utilizou esse tipo de estratégia.

Ao identificar que uma grande proporção dos estudantes do curso de Educação Física atinge os níveis recomendados de prática de atividade física para benefícios à saúde, os resultados desta revisão vão ao encontro do estudo de Silva et a ${ }^{16}$. Os autores relataram que os estudantes de Educação Física foram os que apresentaram a maior prevalência de atividade física (90\%) quando comparados a discentes de cursos das áreas biológicas e de saúde. A alta prevalência do nível adequado de atividade física em universitários do curso de Educação Física pode estar condicionado ao discurso de conscientização dos benefícios da atividade física presente no curso, aos componentes curriculares associados a um forte caráter prático, e ao fato de pessoas que conservam um estilo de vida ativo se identificarem com o curso no momento da opção pela carreira profissional ${ }^{18}$.

Ainda que todos os estudos incluídos classifiquem mais de $74 \%$ dos estudantes como fisicamente ativos, já se tem claro que diferentes fatores podem influenciar na variação destes resultados, como aspectos sociais e determinantes demográficos e ambientais ${ }^{36}$. De acordo com Joly et al. ${ }^{37}$, é possível classificar as mudanças de comportamento em universitários da seguinte forma: acadêmicas, sociais, pessoais e vocacionais. Dessa forma, o comportamento e o estilo de vida dos estudantes são influenciados pelo ritmo e sistematização das aulas, as novas formas de relacionamento, a construção de identidade e personalidade, assim como pelo desenvolvimento profissional experimentado.

A maioria dos estudos (75\%) vinculados a esta revisão identificou os homens como sendo mais ativos que as mulheres. Tal resultado vai ao encontro da literatura, a qual demonstra que os homens praticam mais atividade física, mesmo quando considerada a população universitária ${ }^{38,39}$. Devido a isso, deve-se considerar abordagens diferenciadas para estímulo à prática de atividade física nesses grupos ${ }^{40,41}$.

Todos os estudos avaliaram o nível de atividade física dos estudantes por meio do IPAQ. O IPAQ tem sua validade e reprodutibilidade comparadas com as de outros instrumentos já aceitos e utilizados internacionalmente ${ }^{41}$, além de sua fácil aplicabilidade. Apesar disso, a utilização desse instrumento não garante a com- parabilidade dos níveis de atividade física encontrados devido às diferenças existentes entre as versões curta $\mathrm{e}$ longa. Segundo Hallal et al..$^{43}$, o IPAQ versão curta tende a fornecer respostas imprecisas por avaliar os quatro domínios da atividade física de forma conjunta e, portanto, deve ter seu uso evitado, exceto no caso de manutenção da consistência e/ou vigilância quando já tenha sido utilizado anteriormente em coleta de baseline.

Quanto à região onde os artigos foram conduzidos, é notório que a quantidade de investigações científicas nas regiões norte e centro-oeste do Brasil são menores quando comparadas as demais regiões do país ${ }^{44}$. Isso não é diferente quando se trata de estudos sobre a prática de atividade física, especialmente entre estudantes. No presente estudo, nenhuma pesquisa foi encontrada nas regiões supracitadas. Estudos devem continuar sendo conduzidos em todas regiões do país, em especial nas regiões Norte e Centro-oeste, de forma a identificar possíveis diferenças socioculturais dessas regiões as quais podem interferir diretamente nos níveis de atividade física da população ${ }^{45}$.

A incorporação de um estilo de vida fisicamente ativo pode incrementar os desdobramentos da atuação profissional em Educação Física quando focada nos desafios do combate à inatividade física e seus desfechos hostis à saúde pública e a qualidade de vida da sociedade contemporânea. Sendo assim, é importante destacar a coerência dos resultados com os próprios objetos de conhecimento da profissão, quando a formação profissional de quem atua por meio e em função do movimento humano, se apresenta de forma aliada a sujeitos fisicamente ativos.

Concluindo, os estudantes do curso de Educação Física se mostraram uma população predominantemente ativa, considerando os ambientes universitários estudados no Brasil. Apesar de demonstrar que os estudantes de Educação Física são ativos, a perspectiva é de que a totalidade desses discentes se encaixe nessa condição visto os benefícios da prática para sua saúde e o exemplo que os mesmos devem dar para a população da importância da vida ativa. Estímulo ao deslocamento ativo e a oportunidade da realização de atividades físicas no período de lazer devem ser estimuladas. A disponibilização de materiais e dos espaços para prática de atividades ginásticas, musculação, entre outras, além da organização de jogos nos intervalos de aula e período extra-classe são algumas das alternativas que podem ser utilizadas pelos gestores das unidades acadêmicas para o aumento do nível de atividade física entre es- 
tudantes do curso de Educação Física e demais cursos universitários.

Além disso, lacunas investigatórias importantes como a escassez de estudos longitudinais, falta de abordagens que considerem várias dimensões (pessoais, psicossociais e ambientais) para examinar o estilo de vida relacionado à atividade física nessa população; e medidas dos níveis de atividade física hegemonicamente subjetivas, foram detectadas. Sugere-se a realização de novos estudos a fim de fortalecer e expandir o corpo de evidências sobre essa temática, em especial nas regiões Norte e Centro-oeste do país, as quais ainda sofrem com a falta de investigações científicas. Reforça-se também a realização de estudos longitudinais e com a utilização de medidas diretas, com o uso de acelerômetros, aliadas a questões subjetivas de forma a melhor compreender o comportamento da atividade física desses indivíduos durante e após a conclusão de sua graduação.

\section{Conflito de interesse}

Os autores declaram não haver conflito de interesses.

\section{Contribuição dos autores}

Silva VT, Silva LR, Oliveira VGB e Ramson BP participaram da concepção do estudo, coleta de dados e redação do estudo. Caputo EL e Silva MC participaram da redação do estudo.

\section{Agradecimentos}

O presente trabalho foi realizado com apoio da Coordenação de Aperfeiçoamento de Pessoal de Nível Superior - Brasil (CAPES) - Código de Financiamento 001.

\section{Referências}

1. Lee IM, Shiroma EJ, Lobelo F, Puska P,Blair SN, Katzmarzyk PT. Effect of physical inactivity on major non-communicable diseases worldwide: an analysis of burden of disease and life expectancy. Lancet. 2012;380(9838):219-29.

2. Bauman A. Updating the evidence that physical activity is good for health - an epidemiological review 2000-2003.J Sci Med Sport. 2004;7(1):6-19.

3. Hallal PC, Andersen LB, Bull FC, Guthold R, Haskell W, Ekelund U. Global physical activity levels: surveillance progress, pitfalls, and prospects. Lancet. 2012;380(9838):247-57.

4. Sallis JF, Cerin E, Conway TL, Adams MA, Frank LD, Pratt $\mathrm{M}$, et al. Physical activity in relation to urban environments in 14 cities worldwide: a cross-sectional study. Lancet. 2016;387(10034):2207-17.

5. Guthold R, Stevens GA, Riley LM, Bull FC. Worldwide trends in insufficient physical activity from 2001 to 2016: a pooled analysis of 358 population-based surveys with 1.9 million participants. Lancet Glob Health. 2018;6:e:1077-86.

6. IBGE. Pesquisa Nacional de Amostra por Domicílio. Práticas de Esporte e Atividade Física. 2015. Rio de Janeiro, 2017. Disponível em: (https://biblioteca.ibge.gov.br/visualizacao/ livros/liv100364.pdf)
7. Oehlschlaeger MHK, Pinheiro RT, Horta B, Gelatti C, San'Tana P. Prevalência e fatores associados ao sedentarismo em adolescentes de área urbana. Rev Saúde Pública. 2004;38(2):157-63.

8. Silva DAS, Silva RJS. Padrão de atividade física no lazer e fatores associados em estudantes de Aracaju-SE. Rev Bras Ativ Fís Saúde. 2008;13(2):94-101.

9. Guerra PH, Farias Junior JC, Florindo AA. Comportamento sedentário em crianças e adolescentes brasileiros: revisão sistemática. Rev Saúde Pública. 2016;50(9):1-15.

10. Azevedo MR, Araújo CL, Silva MCD, Hallal PC. Tracking of physical activity from adolescence to adulthood: a populationbased study. Rev Saúde Pública. 2007; 41(1):69-75.

11. Azevedo MR, Menezes AM, Assunção MC, Gonçalves $H$, Arumi I, Horta BL, et al. Tracking of physical activity during adolescence: the 1993 Pelotas Birth Cohort, Brazil. Rev Saúde Pública. 2014;48(6):925-30.

12. Nahas MV. Atividade física, Saúde e Qualidade de Vida. Londrina: Midiograf; 2006

13. Baer JS. Student factors: understanding individual variation in college drinking. J Stud Alcohol Suppl. 2002;(14):40-53.

14. Franca CD, Colares V. Estudo comparativo de condutas de saúde entre universitários no início e no final do curso. Rev Saúde Pública, 2008;42(3):420-7.

15. Salve MGC. A prática de atividade física: estudo comparativo entre alunos de graduação da UNICAMP. Motriz. 2008;4(3):41-7.

16. Silva GFS, Bergamaschine R, Rosa M, Melo C, Miranda $\mathrm{R}$, Bara Filho M. Avaliação do nível de atividade física de estudantes de graduação das áreas saúde/biológica. Rev Bras Med Esporte 2007;13(1):39-42

17. Mielke GI, Ramis TR, Habeyche CE, Oliz MM, Tessmer MGS, Azevedo MR, et al. Atividade física e fatores associados em universitários do primeiro ano da Universidade Federal de Pelotas. Rev Bras Ativ Fís Saúde 2010;15(1):57-64.

18. Silva DAS. Nível de atividade física e fatores associados em acadêmicos de educação física de uma universidade pública do nordeste do Brasil. Rev Bras Ativ Fís Saúde 2011;16(3):193-8.

19. Bielemann RM, Karine G, Azevedo MR, Reichert FF. Prática de atividade física no lazer entre acadêmicos de Educação Física e fatores associados. Rev Bras Ativ Fís Saúde. 2007;12(3):65-72.

20. Moher D, Liberati A, Tetzlaff J, Altman DG. Preferred reporting items for systematic reviews and meta-analyses: the PRISMA statement. Ann Intern Med. 2009;151:264-9.

21. Santos CM, Pimenta CAM, Nobre MRC. A estratégia PICO para a construção da pergunta de pesquisa e busca de evidências. Rev Latino-Am Enfermagem. 2007;15(3):508-11.

22. Wells GA, Shea B, O'Connell D, Peterson J, Welch V, Losos $\mathrm{M}$, et al. The Newcastle-Ottawa Scale (NOS) for assessing the quality of nonrandomised studies in meta-analyses. [publicação online]; 2014. [acesso em 27 de abril de 2020]. Disponível em http://www.ohri.ca/programs/clinical_ epidemiology/oxford.asp.

23. Herzog R, Pasquin MJA, Díaz C, Barrio JL, Estrada JM, Gil A. Are healthcare workers' intentions to vaccinate related to their knowledge, beliefs and attitudes? A systematic review. BMC Public Health. 2013; 13:154.

24. Melo AB, Carvalho EM, Sá FGS, Cordeiro JP, Leopoldo AS, Lima-Leopoldo AP. Nível de atividade física dos estudantes de graduação em Educação Física da Universidade Federal do Espírito Santo. J Phys Educ. 2016;27(1):1-12.

25. Rech CR, Araújo EDS, Vanat JDR. Autopercepção da imagem corporal em estudantes do curso de educação física. Rev Bras Educ Fís Esp. 2010;24(2):285-92. 
26. Vargas LM, Redkva PE, Cantorani JRH, Hauser MW, Gutierrez GL. Nível de atividade física de acadêmicos de Educação Física à distância de uma universidade pública. Rev Bras Ciênc Mov. 2015;23(2):40-50.

27. Freitas MP, Silva MC, Bandeira FM, Hallal PC, Rombaldi AJ. Associação entre aptidão cardiorrespiratória e nível de atividade física em adultos jovens. Rev Bras Ativ Fís Saúde. 2013;18(2):260-70.

28. Costa B, Batista G, Leão N, Paes P. Qualidade de vida, estágios de mudança de comportamento e nível de atividade física dos acadêmicos de Educação Física. Rev Bras Ativ Fís Saúde. 2015;20(5):476-82.

29. Miranda VPN, Neves CM, Filgueiras JF, Carvalho PHB, Ferreira MEC. Nível de atividade física e satisfação corporal em estudantes de Educação Física. Rev Bras Ciênc Mov. 2013;21(2):98-105.

30. Lima GO, Mendes BM, Klein SK, Formentin CM, Garlipp DC. Nível de atividade física e risco de desenvolvimento de doenças cardiovasculares em acadêmicos do curso de Educação Física. Rev. Bras. Prescrição e Fisiol do Exerc. 2017;11(68):542-9.

31. Santos TS, Leão OAA, Leite JS, Silva MC. Atividade física em acadêmicos de Educação Física: um estudo longitudinal. Rev Bras Ativ Fís Saúde. 2017;22(1):76-84.

32. Ferrari EP, Petroski EL, Silva DAS. Prevalência de insatisfação com a imagem corporal e fatores associados em universitários de educação física. Trends Psychiatry Psychother. 2013;35(2):119-27.

33. Meira CEV, Andrade RD. Atividade física no lazer, capacidade aeróbia percebida e bem-estar subjetivo de acadêmicos de educação física em diferentes fases do curso. Cad Educ Física e Esporte [Internet]. 2019;17(1):13-21.

34. Monteiro LZ, Varela AR, Lira BA, Souza P, Gomes DJO, Contiero LC, et al. Behavioral health risk profiles of physical education undergraduates. Mot Rev Educ Física [Internet]. 2019;25(4).

35. Berbigier MC, Magalhães CR. Educação nutricional em universitários e estratégias para promoção de saúde institucional: revisão integrativa. Rev Bras Promoç Saúde. 2017;30(2):264-74.
36. Deliens T, Deforche B, Bourdeaudhuij I, Clarys P. Determinants of physical activity and sedentary behaviour in university students: a qualitative study using focus group discussions. BMC Public Health. 2015; 15:201.

37. Joly MCRA, Santos AAA, Sisto FF. Questões do cotidiano universitário. São Paulo: Casa do Psicólogo; 2005. cap. 8, p. 159-77.

38. Colares V, Franca C, Gonzalez E. Condutas de Saúde entre universitários: diferenças entre gêneros. Cad Saúde Pública. 2009; 25(3):521-28.

39. Palma A, Abreu RA, Cunha CA. Comportamentos de risco e vulnerabilidade entre estudantes de educação física. Rev Bras Epidemiol. 2007;10(1):117-26.

40. Lowry R, Galuska DA, Fulton JE, Wechsler H, Kann L, Collins JL. Physical activity, food choice, and weight management goals and practices among U.S. college students. Am J Prev Med. 2000;18:18-27.

41. Davies J, McCrae BP, Frank J, Dochnahl A, Pickering T, Harrison B, et al. Identifying male college students' perceived health needs, barriers to seeking help, and recommendations to help men adopt healthier lifestyles. J Am Coll Health. 2000;48:259-67.

42. Matsudo S, Araújo T, Matsudo V, Andrade D, Andrade E, Oliveira LC, et al. Questionário internacional de atividade física (IPAQ): estudo de validade e reprodutibilidade no Brasil. Rev Bras Ativ Fís Saúde. 2001;6:5-18.

43. Hallal PC, Gomez LF, Parra DC, Lobelo F, Mosquera J, Florindo AA, et al. Lessons learned after 10 years of IPAQ use in Brazil and Colombia. J Phys Act Health. 2010;7(2):259-64.

44. Sidone OJG, Haddad EA, Mena-Chalco JP. A ciência nas regiões brasileiras: evolução da produção e das redes de colaboração científica. Transinformação. 2016;28(1):15-32.

45. Santos CM, Barbosa JMV, Cheng LA, Júnior RSW, Barros MVG. Atividade Física no contexto dos deslocamentos: Revisão sistemática dos estudos epidemiológicos realizados no Brasil. Rev Bras Ativ Fís Saúde. 2009;14(1):15-22.

Recebido: $27 / 08 / 2020$

Aprovado: $15 / 04 / 2021$

Como citar este artigo:

Silva VT, Silva LR, Oliveira VGB, Ramson BP, Caputo EL, Silva MC. Nivel de atividade física de estudantes de educação física no Brasil: uma revisão sistemática. Rev Bras Ativ Fís Saúde. 2021;26:e0197. DOI: 10.12820/rbafs.26e0197 